\title{
MicroRNA-target pairs in the rat kidney identified by microRNA microarray, proteomic, and bioinformatic analysis
}

\author{
Zhongmin Tian, ${ }^{1,2}$ Andrew S. Greene, ${ }^{1,2}$ Jennifer L. Pietrusz, ${ }^{1}$ Isaac R. Matus, ${ }^{2}$ \\ and Mingyu Liang ${ }^{1,3}$ \\ ${ }^{1}$ Department of Physiology, Medical College of Wisconsin, Milwaukee, Wisconsin 53226, USA; ${ }^{2}$ Biotechnology and Biomedical \\ Engineering Center, Medical College of Wisconsin, Milwaukee, Wisconsin 53226, USA
}

\begin{abstract}
Mammalian genomes contain several hundred highly conserved genes encoding microRNAs. In silico analysis has predicted that a typical microRNA may regulate the expression of hundreds of target genes, suggesting miRNAs might have broad biological significance. A major challenge is to obtain experimental evidence for predicted microRNA-target pairs. We reasoned that reciprocal expression of a microRNA and a predicted target within a physiological context would support the presence and relevance of a microRNA-target pair. We used microRNA microarray and proteomic techniques to analyze the cortex and the medulla of rat kidneys. Of the 377 microRNAs analyzed, we identified 6 as enriched in the renal cortex and 11 in the renal medulla. From $\sim 2100$ detectable protein spots in two-dimensional gels, we identified 58 proteins as more abundant in the renal cortex and 72 in the renal medulla. The differential expression of several microRNAs and proteins was verified by real-time PCR and Western blot analyses, respectively. Several pairs of reciprocally expressed microRNAs and proteins were predicted to be microRNA-target pairs by TargetScan, PicTar, or miRanda. Seven pairs were predicted by two algorithms and two pairs by all three algorithms. The identification of reciprocal expression of microRNAs and their computationally predicted targets in the rat kidney provides a unique molecular basis for further exploring the biological role of microRNA. In addition, this study establishes a differential profile of microRNA expression between the renal cortex and the renal medulla and greatly expands the known differential proteome profiles between the two kidney regions.
\end{abstract}

[Supplemental material is available online at www.genome.org.]

MicroRNAs are a class of endogenous, conserved, small regulatory RNA, the discovery of which has been hailed as one of the most important breakthroughs in biology in recent years (Couzin 2002; Dennis 2002). MicroRNA is encoded by specific genes in plant and animal genomes and may act primarily through binding to the 3' untranslated region of target mRNA and suppressing protein translation (Ambros 2004; Bartel 2004; He and Hannon 2004; Kim 2005). In some cases, microRNA can also reduce the abundance of target mRNA (Bagga et al. 2005; Lim et al. 2005). Since microRNA exerts its biological effects through suppression of target genes, it is necessary to identify microRNA-target pairs to understand the biological significance of specific microRNAs.

An interesting characteristic of microRNA is that it only requires partial complementarities with its target sequence. As a result, a microRNA could potentially target multiple genes. One could predict which genes might be targeted by a microRNA based on the sequence characteristics of known microRNA-target pairs (Rajewsky 2006). This type of in silico analysis has predicted several hundred thousand microRNA-target pairs in human, rat, and mouse (John et al. 2004; Lewis et al. 2005). Several thousand protein-coding genes in mammalian genomes have been predicted to be targets of a few hundred known microRNAs. This suggests that microRNAs may play an extremely broad and important role in biological regulation.

The fact that only partial sequence complementarities are needed, however, makes it challenging to ascertain the presence

\section{${ }^{3}$ Corresponding author.}

E-mail mliang@mcw.edu; fax (414) 456-6546.

Article published online before print. Article and publication date are at http:// www.genome.org/cgi/doi/10.1101/gr.6587008. of a microRNA-target pair (Krutzfeldt et al. 2006). Only a few dozen microRNA-target pairs have been experimentally validated in human, rat, or mouse (see http://microrna.sanger.ac.uk/ cgi-bin/targets/v4/known_targets.pl), despite the prediction of several hundred thousand. Experiments to validate the presence and relevance of a microRNA-target pair are laborious and timeconsuming. It would be highly valuable to identify those predicted microRNA-target pairs that are more likely to be present and relevant.

We reasoned that reciprocal expression of a microRNA and a predicted target within a physiological context would support the presence and physiological relevance of a predicted microRNA-target pair. We used microRNA microarray and proteomic techniques to analyze the cortex and the medulla of rat kidneys. The renal cortex and the renal medulla are functionally and structurally distinct, yet closely related, providing an interesting context for identifying possible microRNA-target pairs that may be physiologically relevant. Reciprocal expression of several pairs of microRNAs and their computationally predicted targets was identified in the rat kidney, a differential profile of microRNA expression in the renal cortex and medulla was established, and the known differential proteome profiles in the two kidney regions were greatly expanded.

\section{Results}

MicroRNA expression profiles in the renal cortex and the renal medulla

The 377 microRNAs analyzed included 170 , or $45 \%$, that were known rat microRNAs. In the renal cortex 110 microRNAs were 
considered detectable, while 71 were detectable in the renal medulla. Among the detectable microRNAs in the renal cortex and the renal medulla, $62 \%$ and $79 \%$, respectively, were known rat microRNAs. The four most abundant microRNAs in both the renal cortex and the renal medulla, according to backgroundsubtracted and normalized fluorescent intensities, were rno-let7c, rno-miR-26a, rno-let-7b, and rno-let-7a. Absolute abundance levels, however, should be interpreted cautiously since fluorescent intensities might be affected by other factors, such as variations in probe concentrations and the efficiency of printing pins.

We found six microRNAs to be preferentially expressed in the renal cortex, and 11 in the renal medulla (Fig. 1). The false positive rate, according to a permutation approach described previously (Morrison et al. 2004), was 3.9\%. The degree of enrichment of specific microRNAs in one kidney region compared with the other ranged from 1.5-fold to more than 100-fold (Fig. 1). Of the 17 differentially expressed microRNAs, 15 were known rat microRNAs. Hsa-miR-450 (currently hsa-miR-450a) is a human microRNA identical to the rat microRNA rno-miR-450, which was not represented in the array, except the last nucleotide.

We selected three microRNAs, rno-miR-192, rno-miR-194, and rno-miR-27b, which represented varying degrees of differential expression, for further analysis using modified real-time PCR $(n=6)$. All three microRNAs were verified as exhibiting statistically significant differential expression between the two kidney regions (Fig. 2). The modified real-time PCR analysis, compared with microarray, indicated smaller fold changes for two microRNAs and greater fold changes for one.

\section{Proteome profiles in the renal cortex and the renal medulla}

On average, 2140 spots were detected in each of the four threecolor gels, a representative picture of which is shown in Supplemental Figure 1. Of 629 spots that were considered differentially expressed between the renal cortex and the renal medulla, 409 were picked for mass spectrometry analysis. Protein identities were obtained for 279 spots, or $68 \%$ of those picked. One hundred ten of the identified spots were more abundant in the renal

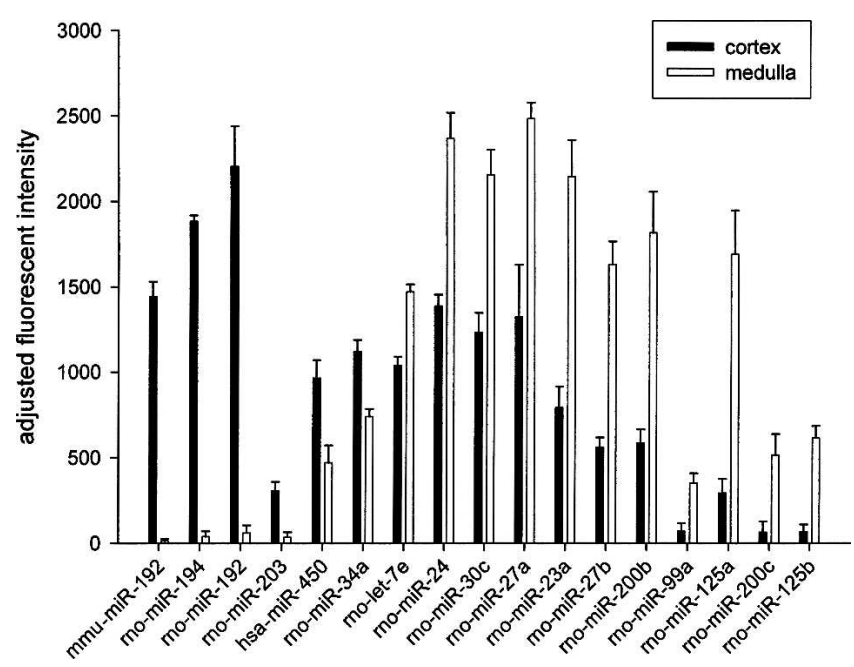

Figure 1. MicroRNAs differentially expressed between the renal cortex and the renal medulla in Sprague-Dawley rats. Expression levels of 377 microRNAs were measured by microRNA microarrays $(n=4)$. Differentially expressed microRNAs, six enriched in the cortex and 11 in the medulla, are shown. MicroRNAs are ranked, from left to right, by cortex/ medulla ratios.

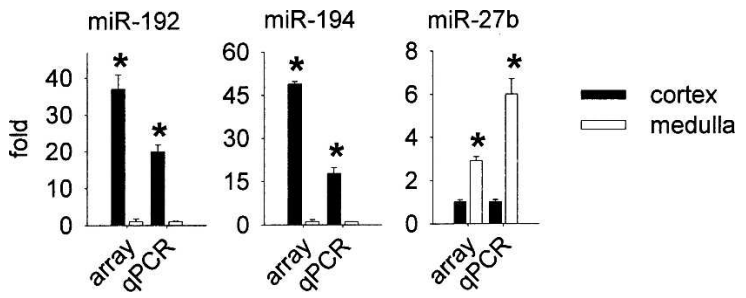

Figure 2. Verification of microRNA differential expression by real-time $P C R$. Fold differences between the renal cortex and the renal medulla measured by microarray (array) and real-time PCR (qPCR) are shown. $n=6 ;\left(^{*}\right)$ significantly different from the other kidney region.

cortex, and 169 were more abundant in the renal medulla. The spot ID, common protein name, $P$-value, ratio between the two kidney regions, UniProt ID, gene symbol, theoretical isoelectric point (pI) and molecular weight, gel-based pI and molecular weight, and sequence coverage, score and Expect values from the Mascot analysis for each of these spots are described in Supplemental Tables 1 and 2. The location of each spot on the gel is shown in Supplemental Figures 2 and 3.

We consider a protein as having different abundance levels in the two kidney regions if the protein was identified in a single differentially expressed spot. A protein might be identified in multiple differentially expressed spots. All spots representing a protein should be consistently more abundant in one kidney region than the other for the protein to be considered having different abundance levels in the two kidney regions. On the basis of these considerations, 58 proteins were considered more abundant in the cortex and 72 in the medulla. There were four proteins, F1-ATPase beta chain (Atp5b), F1-ATPase alpha chain isoform 1 (Atp5a1), beta actin (Actb), and lamin A (Lmna), in which some spots of a protein were more abundant in one kidney region while other spots of the same protein were more abundant in the other region. These four proteins might be modified or processed differently in the two regions.

We performed Western blot analysis for four of the identified proteins, copper-zinc containing superoxide dismutase (Sod1), catalase (Cat), fumarase (Fh1), and heterogeneous nuclear ribonucleoprotein $\mathrm{K}$ (Hnrpk). The differential expression of all four proteins was verified (Fig. 3).

\section{Possible microRNA-target pairs supported by sequence characteristics and reciprocal expression patterns}

We used TargetScan, PicTar, and miRanda to predict the targets of the differentially expressed microRNAs. In the 16 differentially expressed microRNAs (see Fig. 1), miR-27a and miR-27b, miR$125 \mathrm{a}$ and miR-125b, and miR-200b and miR-200c have nearly identical sequences and identical or nearly identical predicted targets according to all three algorithms. These microRNAs were consolidated, resulting in 13 microRNAs that were considered in the subsequent informatic analysis.

Thirteen of 798 reciprocally expressed pairs of microRNAs and proteins were computationally predicted to be microRNAtarget pairs according to TargetScan (Table 1). The proportion of matches $\left(\mathrm{P}_{\mathrm{r}}\right), 0.0163$, was significantly greater than what can be expected from random microRNAs $\left(\mathrm{P}_{\mathrm{m}}=0.0050, Z\right.$-test $\left.P<0.05\right)$ and tended to be greater than coexpression $\left(\mathrm{P}_{\mathrm{C}}=0.0071\right.$, $P=0.131$ ) (Fig. 4A). When PicTar was used, $\mathrm{P}_{\mathrm{r}}(0.0110)$ tended to be greater than $\mathrm{P}_{\mathrm{m}}(0.0026, P=0.096)$ and $\mathrm{P}_{\mathrm{c}}(0.0038, P=0.179)$. $P_{r}$ was slightly but not significantly higher than $P_{m}$ or $P_{c}$ when 

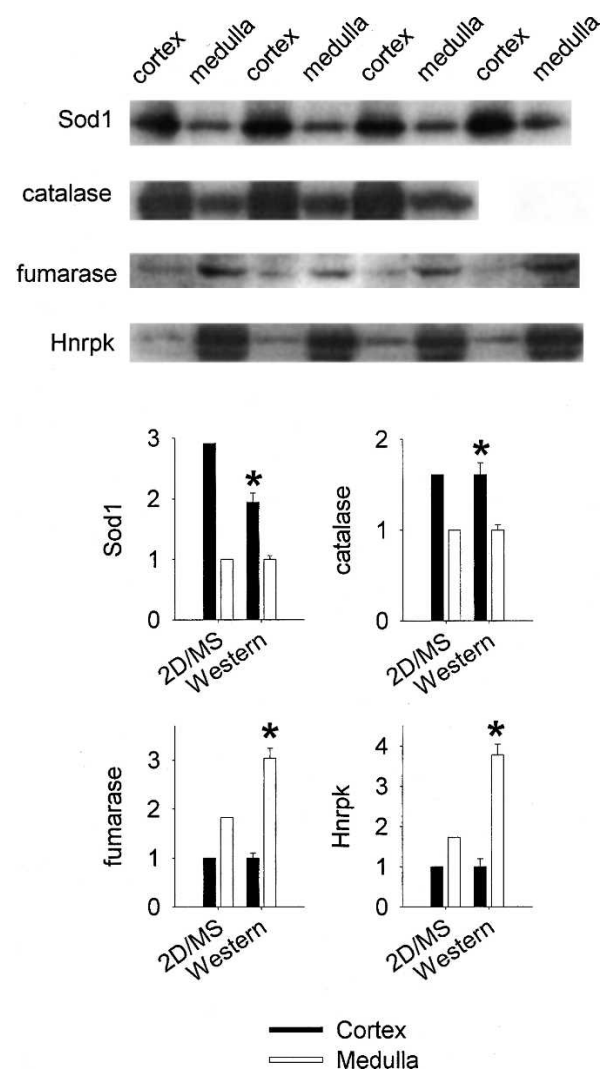

Figure 3. Verification of protein differential expression by Western blotting. Western blots and fold differences between the renal cortex and the renal medulla measured by proteomic techniques (2D/MS) and Western blotting (Western) are shown. Band densities in Western blots were normalized to Coomassie blue staining. Note that the differences found in the proteomic analysis were statistically significant based on four rats, although error bars are not provided. (Sod1) Copper-zinc containing superoxide dismutase; (Hnrpk) heterogeneous nuclear ribonucleoprotein K. $n=3-4 ;\left(^{*}\right) P<0.05$ vs. the other kidney region.

miRanda was used. Similar statistical results were obtained when only microRNAs with absolute $\log _{2}$ ratios $>1$ were considered.

In total, 52 pairs of reciprocally expressed microRNAs and proteins were predicted by at least one of the three algorithms to be microRNA-target pairs (Table 1). Two pairs were predicted by all three algorithms, while seven pairs were predicted by two algorithms. TargetScan and PicTar had an overlap of $67 \%$. The overlap between TargetScan and miRanda and between PicTar and miRanda was $14 \%$ and $8 \%$, respectively. A summary of the reciprocal expression and the match with computational predictions is shown in Supplemental Figure 4.

The fold changes of the 42 proteins in Table 1 tended to be greater than those of the proteins that were differentially expressed but not predicted to be targets of microRNA, but the difference did not reach statistical significance (absolute $\log _{2}$ ratio, $1.33 \pm 0.09$ vs. $1.20 \pm 0.04, P=0.127)$. Of the 42 genes in Table 1, eight were predicted targets of two microRNAs each, and one was a predicted target of three microRNAs. No significant difference was observed between the fold changes of these nine proteins and the proteins that were predicted targets of one microRNA each. Four genes have two binding sites for a microRNA. There was no significant difference between the fold changes of these four proteins and the remaining proteins in Table 1.
One of the predicted microRNA-target pairs, miR-450 and HNRPK, was further tested in HK-2 cells. The pair was selected in part because of our ongoing interest in HNRPK. As shown in Figure 4B, blocking endogenous miR-450 with anti-miR-450 slightly but significantly increased the protein expression level of HNRPK. Increasing cellular contents of miR-450 with pre-miR450 significantly decreased the protein level of HNRPK. mRNA levels of $H N R P K$ were not affected. Similar results were obtained in human umbilical vein endothelial cells, in which anti-miR450 increased the protein level of HNRPK by $23 \pm 4 \%$, while pre-miR-450 decreased it by $28 \pm 3 \%(n=4, P<0.05$ vs. control oligonucleotides). These data provided evidence supporting the regulation of HNRPK by miR-450 that was suggested by the microarray, proteomic, and bioinformatic analysis.

\section{Discussion}

The present study has made three contributions. First, we have identified reciprocal expression of several microRNAs and their predicted targets in the rat kidney. Second, we have established a differential profile of microRNA expression between the renal cortex and medulla. Third, we have greatly expanded the known differential proteome profiles in the two kidney regions.

The present study provided evidence of reciprocal expression for a large set of computationally predicted microRNAtarget pairs. Establishing the presence and relevance of any one microRNA-target pair often requires a major, time-consuming study, in part due to the lack of high-confidence candidates. Computational prediction of microRNA-target pairs considers sequence characteristics and provides a valuable starting point. Demonstration of a reciprocal expression pattern is a significant step beyond computational prediction and substantially increases the level of confidence on calling the presence of a microRNA-target pair. The large set of reciprocally expressed pairs of microRNAs and predicted targets that we identified could significantly accelerate the study of the biological significance of microRNA.

The possible microRNA-target pairs identified in the present study exhibited reciprocal expression under physiological conditions. The traditional approach to identifying possible microRNA-target pairs is to experimentally inhibit or overexpress a specific microRNA and examine subsequent changes in gene or protein expression (Lim et al. 2005; Krutzfeldt et al. 2006; Vinther et al. 2006; Wang and Wang 2006). Both our approach and the traditional approach rely on sequence characteristics to distinguish direct interactions between a microRNA and its targets from secondary effects. The advantage of our approach is the reciprocal expression patterns we observed were present in undisturbed in vivo conditions, supporting not only the presence, but also the physiological relevance, of the identified microRNAtarget pairs. Our approach also allows the investigation of many microRNAs simultaneously. The advantage of the traditional approach is that the experimental alterations of a particular microRNA are presumably specific and can be well controlled.

The proportion of reciprocally expressed pairs of microRNAs and proteins that matched computationally predicted microRNA-target pairs was higher or tended to be higher than the proportions found with coexpression or randomly selected microRNAs. This is consistent with the finding that, in Drosophila, microRNAs and their targets tended to be expressed in neighboring tissues rather than in the same tissue (Stark et al. 2005).

\section{Genome Research} www.genome.org 
MicroRNA-target pairs in the rat kidney

Table 1. Reciprocally expressed microRNAs and computationally predicted targets

\begin{tabular}{|c|c|c|c|c|c|c|c|}
\hline miRNA & $\begin{array}{c}\operatorname{miRNA} \\
\log _{2}(M / C)^{a}\end{array}$ & $\begin{array}{l}\text { Target } \\
\text { symbol }\end{array}$ & Target common name & $\begin{array}{c}\text { Protein } \\
\log _{2}(M / C)\end{array}$ & TargetScan & PicTar & miRanda \\
\hline miR-194 & -5.61 & Bckdha & Branched chain keto acid dehydrogenase E1, alpha & 0.97 & Yes & Yes & \\
\hline miR-194 & -5.61 & $C k b$ & Creatine kinase (EC 2.7.3.2) chain $B$ & 0.82 & & & Yes \\
\hline miR-194 & -5.61 & Myh6 & Myosin alpha heavy chain & 0.84 & & & Yes \\
\hline miR-194 & -5.61 & Pdhb & Pyruvate dehydrogenase beta & 1.05 & Yes & Yes & Yes $(2)^{\mathrm{b}}$ \\
\hline miR-192 & -5.21 & Fabp3 & Fatty acid binding protein 3 & 0.89 & Yes & & \\
\hline miR-192 & -5.21 & Ldhb & L-Lactate dehydrogenase B chain (EC 1.1.1.27) & 1.48 & & & Yes \\
\hline miR-203 & -3.17 & Aco2 & Aconitase 2 , mitochondrial & 1.21 & Yes & Yes & Yes \\
\hline miR-203 & -3.17 & Dars & Aspartate-tRNA ligase (EC 6.1.1.12) & 0.67 & & & Yes \\
\hline miR-203 & -3.17 & Eif4a1 & Eukaryotic translation initiation factor $4 \mathrm{~A} 1$ & 1.12 & & & Yes \\
\hline miR-203 & -3.17 & Gdi1 & GDP dissociation inhibitor 1 & 2.76 & & Yes & \\
\hline miR-450 & -1.04 & Anxa5 & Annexin V & 1.6 & & NA & Yes \\
\hline miR-450 & -1.04 & Capza2 & F-actin capping protein alpha-2 subunit & 0.81 & & NA & Yes \\
\hline miR-450 & -1.04 & Hnrpk & Heterogeneous nuclear ribonucleoprotein $\mathrm{K}$ & 0.79 & Yes & NA & \\
\hline miR-450 & -1.04 & Tpm3_v1 & Tropomyosin 5 & 1.06 & & NA & Yes \\
\hline miR-450 & -1.04 & Vim & Vimentin & 1.07 & & NA & Yes \\
\hline miR-34a (34) & -0.6 & Aldoa & Aldolase A, fructose-bisphosphate & 2.42 & Yes & Yes & \\
\hline miR-34a & -0.6 & Hspa1a & DnaK-type molecular chaperone HSP70 & 1.93 & & & Yes \\
\hline miR-34a (34) & -0.6 & $\mathrm{Vcl}$ & Vinculin & 1.72 & Yes & Yes & \\
\hline let-7e & 0.5 & Acads & Acyl-CoA dehydrogenase (EC 1.3.99.3) short-chain & -0.82 & & & Yes $(2)^{b}$ \\
\hline let-7e & 0.5 & Selenbp 1 & Selenium binding protein 2 & -1.25 & & & Yes \\
\hline miR-24 & 0.77 & $G p \times 3$ & Glutathione peroxidase 3 & -2.35 & Yes & Yes & \\
\hline miR-30c & 0.81 & Cndp2 & Nonspecific dipeptidase & -1.14 & & & Yes \\
\hline miR-30c (30-5p) & 0.81 & Hspa5 & Heat shock 70kDa protein 5 & -1.65 & Yes & & Yes \\
\hline miR-30c (30-5p) & 0.81 & Idh1 & Isocitrate dehydrogenase $1(\mathrm{NADP}+)$, soluble & -1.04 & Yes & & \\
\hline $\mathrm{miR}-30 \mathrm{c}$ & 0.81 & Vdac1 & Voltage-dependent anion-selective channel protein 1 & -0.81 & & & Yes \\
\hline $\operatorname{miR}-27 a /-27 b$ & 0.91 & Acadm & Acyl-CoA dehydrogenase (EC 1.3.99.3), medium-chain & -0.71 & & & Yes \\
\hline $\operatorname{miR}-27 a /-27 b$ & 0.91 & Acads & Acyl-CoA dehydrogenase (EC 1.3.99.3) short-chain & -0.82 & & & Yes \\
\hline $\operatorname{miR}-27 a /-27 b$ & 0.91 & Cyb5 & Cytochrome b5 & -1.84 & & & Yes \\
\hline miR-27a/-27b & 0.91 & Msra & Peptide methionine sulfoxide reductase & -1.39 & & & Yes \\
\hline miR-23a & 1.44 & Abhd $14 b$ & Abhydrolase domain containing $14 \mathrm{~b}$ & -0.76 & & & Yes \\
\hline miR-23a & 1.44 & Agxt2 & Beta-alanine-pyruvate aminotransferase & -2.19 & & & Yes \\
\hline miR-23a & 1.44 & Aldh9a1 & 4-Trimethylaminobutyraldehyde dehydrogenase & -1.17 & & & Yes \\
\hline miR-23a (23) & 1.44 & Atp6v1b2 & ATPase, $\mathrm{H}+$ transporting, lysosomal $56 / 58 \mathrm{kDa}, \mathrm{V} 1 \mathrm{~B} 2$ & -0.96 & Yes & & \\
\hline miR-23a (23) & 1.44 & Atp6r1e1 & ATPase, $\mathrm{H}+$ transporting, lysosomal $31 \mathrm{kDa}, \mathrm{V} 1 \mathrm{E} 1$ & -0.84 & Yes & & Yes \\
\hline miR-23a & 1.44 & Cryl1 & Lambda-crystallin homolog & -1.88 & & & Yes \\
\hline miR-23a & 1.44 & Gatm & L-Arginine-glycine amidinotransferase & -1.24 & & & Yes \\
\hline miR-23a (23) & 1.44 & Idh1 & Isocitrate dehydrogenase $1(\mathrm{NADP}+)$, soluble & -1.04 & Yes & Yes & \\
\hline miR-99a & 2.31 & Gatm & L-Arginine-glycine amidinotransferase & -1.24 & & & Yes \\
\hline miR-125a/-125b & 2.53 & Acads & Acyl-CoA dehydrogenase (EC 1.3.99.3) short-chain & -0.82 & & & Yes \\
\hline miR-125a/-125b & 2.53 & Agxt2 & Beta-alanine-pyruvate aminotransferase & -2.19 & & & Yes \\
\hline miR-125a/-125b & 2.53 & Cndp2 & Nonspecific dipeptidase & -1.14 & & & Yes \\
\hline miR-125a/-125b & 2.53 & Dpp4 & Dipeptidyl peptidase 4 (EC 3.4.14.5) & -1.44 & & & Yes \\
\hline miR-125a/-125b & 2.53 & Gss & Glutathione synthase (EC 6.3.2.3) & -0.86 & & & Yes \\
\hline miR-125a/-125b & 2.53 & Hspa5 & Heat shock $70 \mathrm{kDa}$ protein 5 & -1.65 & & & Yes $(2)^{b}$ \\
\hline miR-125a/-125b & 2.53 & Mpst & 3-Mercaptopyruvate sulfurtransferase (EC 2.8.1.2) & -1.86 & & & Yes \\
\hline miR-125a/-125b & 2.53 & Acadm & Acyl-CoA dehydrogenase (EC 1.3.99.3), medium-chain & -0.71 & & & Yes \\
\hline miR-125a/-125b & 2.53 & Aldh2 & Aldehyde dehydrogenase, mitochondrial (EC 1.2.1.3) & -1.27 & & & Yes \\
\hline $\mathrm{miR}-200 \mathrm{c} /-200 \mathrm{~b}$ & 3.01 & Acy 1 & Kidney aminoacylase 1 & -1.58 & & & Yes \\
\hline $\mathrm{miR}-200 \mathrm{c} /-200 \mathrm{~b}$ & 3.01 & Aldh9a1 & 4-Trimethylaminobutyraldehyde dehydrogenase & -1.17 & & & Yes $(2)^{b}$ \\
\hline $\mathrm{miR}-200 \mathrm{c} /-200 \mathrm{~b}$ & 3.01 & Cyb5 & Cytochrome b5 & -1.84 & & & Yes \\
\hline $\operatorname{miR}-200 c /-200 b$ & 3.01 & Got1 & Aspartate transaminase (EC 2.6.1.1) & -2.71 & & & Yes \\
\hline $\mathrm{miR}-200 \mathrm{c} /-200 \mathrm{~b}$ & 3.01 & Lap3 & Leucine aminopeptidase 3 & -1.01 & & & Yes \\
\hline
\end{tabular}

In TargetScan 3.1, searches for miR-34a, miR-30c, and miR-23a returned miR-34, miR-30-5p, and miR-23, respectively. miR-450 was not available in PicTar.

${ }^{a} \log _{2}(M / C), \log _{2}$ ratio of medulla over cortex

bYes (2) indicates that the target has two predicted binding sites for the microRNA.

Wide-spread reciprocal expression and, in some cases, coexpression, of microRNAs and their target mRNAs have been reported in mammalian species (Farh et al. 2005; Sood et al. 2006; Tsang et al. 2007). The present study demonstrated reciprocal expression of microRNAs and proteins encoded by microRNA target mRNAs. For the few cases of coexpression found in the present study, it would be interesting to examine whether up-regulation of microRNA represents a negative feedback response to upregulation of target proteins. While the proportion statistics appear to suggest that TargetScan and PicTar have fewer false posi- tives than miRanda, validation experiments on a large scale would be needed to systematically compare various target prediction algorithms.

Both the renal cortex and the renal medulla are involved in the general process of tubular transport, contain continuous tubular loops and vasculature, and consist largely of epithelial cells. The two regions differ in several aspects, including filtration and specific transport activities, blood and oxygen supply, and interstitial osmolarity. MicroRNA-target pairs that are reciprocally expressed in the renal cortex and the renal medulla may participate 

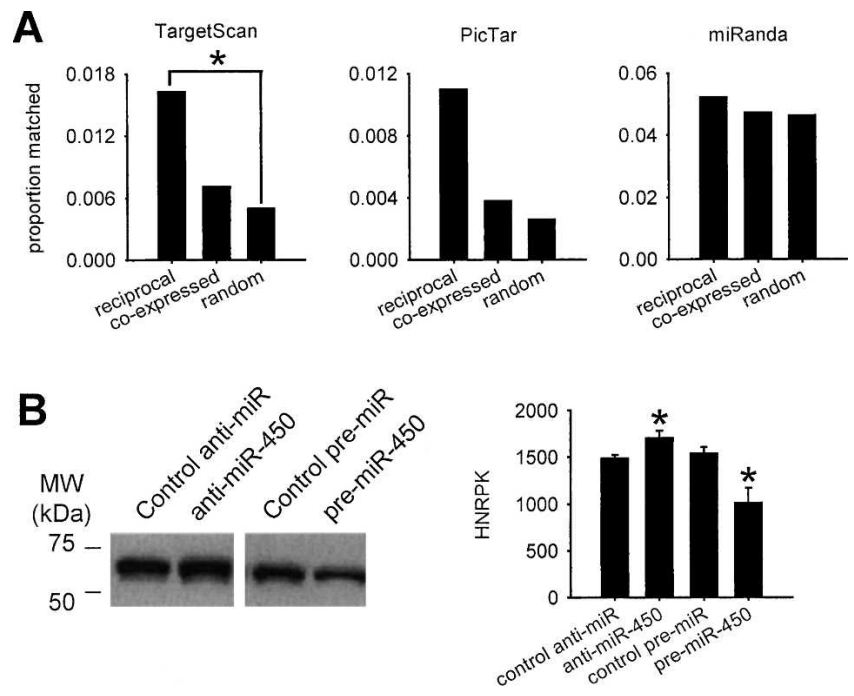

Figure 4. Analysis of reciprocal expression of microRNAs and computationally predicted targets. $(A)$ Proportions of reciprocally expressed or coexpressed microRNA and protein pairs, or pairs of randomly selected microRNAs and differentially expressed proteins, that matched computational prediction using the indicated algorithm are shown. Details of the calculation are described in Methods. $\left(^{*}\right) P<0.05$ (Z-test). (B) AntimiR-450 and pre-miR-450 (50 nM) significantly increased and decreased, respectively, the protein expression level of HNRPK in HK-2 cells. $n=4$; $\left(^{*}\right)$ $P<0.05$ vs. control.

in maintaining the tissue identity or regulating physiological activities in the two kidney regions.

The number of possible microRNA-target pairs identified in the present study was large in the context of the current knowledge of microRNA. However, they likely represent a small subset of all microRNA-target pairs functioning in the kidney. Only a fraction of a tissue proteome can be analyzed by the proteomic techniques used in this study. It is likely that multiple mechanisms are involved in regulating the expression level of a protein. Differences in mRNA levels may offset the effect of a microRNA, resulting in the lack of differential protein expression. Conversely, differential expression of a protein may be due to transcriptional regulation or other mechanisms, not the action of microRNA. Measurement of mRNA levels, however, would not definitively distinguish transcriptional regulation from microRNA actions since microRNA could affect mRNA levels in some cases (Bagga et al. 2005; Lim et al. 2005). In addition, microRNA-target pairs that are present similarly in the two kidney regions would not have been identified in the present study. In other words, a microRNA that is not differentially expressed between the two kidney regions could nonetheless be functionally important in the kidney. Furthermore, microRNA may have indirect effects on protein expression. For instance, downregulation of a protein by a microRNA could lead to upregulation of other proteins that are influenced by the direct target of the microRNA. It should also be acknowledged that currently available predictions of microRNA-target pairs likely do not include all possible microRNA-target pairs.

The present study established the first differential profile of microRNA expression in the renal cortex and the renal medulla. MicroRNA expression profiles in whole kidneys have been reported previously (Liu et al. 2004; Thomson et al. 2004; Naraba and Iwai 2005). The difference between microRNA profiles in the cortex and the medulla was substantial, suggesting a significant role for microRNA in renal physiology. It is interesting to note that miR-192, which we found highly enriched in the cortex, was recently reported to be possibly involved in glomerular injury in diabetic mice (Kato et al. 2007). A complete set of the microRNA microarray data is available in Supplemental Table 3.

The present study greatly expanded the known differential proteome profile in the renal cortex and medulla. A number of groups have performed comparative analysis of renal cortical and medullary proteomes (Witzmann et al. 1998; Arthur et al. 2002). The number of regionally enriched proteins identified in the present study was several times more than previously reported. This was made possible by recently available proteomic techniques. Several proteins we identified were also found in previous studies. Examples include enrichment of sorbitol dehydrogenase (Sord), aldehyde dehydrogenase (Aldh2), alpha $2 \mathrm{u}$ globulin, and isocitrate dehydrogenase (Idh1) in the cortex, and transferrin (Tf), aldose reductase (Akr1b4), and albumin (Alb) in the medulla. Some of the identified proteins might be from residual plasma even though the kidneys were flushed with normal saline. The use of the differential in-gel electrophoresis (DIGE) technique also allowed us to detect small shifts in spot locations and identify a number of proteins that might be modified differently in the two kidney regions. A notable absence in the identified proteins was transporters and signaling molecules, probably due to the limited ability of our proteomic techniques to detect membrane or low-abundance proteins (Hoorn et al. 2005).

The cortex and the medulla each consist of a mix of anatomical structures and cell types, making it difficult to predict the functional significance of some of the identified proteins. Nonetheless, the expanded proteome profile may provide insights into the molecular basis of renal function and facilitate further studies of renal physiology and disease. For example, several key antioxidant proteins, including copper-zinc containing superoxide dismutase (Sod1), catalase (Cat), glutathione synthase (Gss), and glutathione peroxidase 3 (Gpx3), were expressed at higher levels in the renal cortex than in the renal medulla. This expression pattern might contribute to or reflect different redox states in the two kidney regions (Gonzalez-Flecha et al. 1993), which might be relevant to the regulation of arterial blood pressure (Taylor et al. 2006).

The present study points to several exciting directions for future research. Each possible microRNA-target pair we identified is a strong candidate for a major study to definitively confirm the presence of specific microRNA-target interactions. It will be important to examine the functional significance of the identified microRNA-target pairs and regionally enriched proteins in renal physiology and disease. As more microRNA-target pairs are established, we could further improve our ability to predict microRNA targets and better understand the fundamental biology of microRNA.

\section{Methods}

Isolation of microRNA from rat renal cortical and medullary tissues

The kidneys were harvested from four male Sprague-Dawley rats weighing 250-300 g, the renal cortex and the renal medulla were dissected, and total RNA was extracted as we described previously (Liang et al. 2002, 2003; Liang and Pietrusz 2007). MicroRNAs were enriched using YM-30 columns (nucleotide length cutoff 60 for single-stranded, and 50 for double-stranded), similar to a pre- 
viously reported method (O'Donnell et al. 2005). The enrichment procedure removed $99 \%$ of $5 \mathrm{~S}$ rRNA according to real-time PCR analysis.

\section{Construction and hybridization of microRNA microarray}

A microRNA probe set representing 377 microRNAs and several controls (Ambion) were resuspended in the Schott Spot printing solution (Schott) to obtain a final concentration of $\sim 25 \mu \mathrm{M}$. The amine-modified probes were printed in triplicate on Nexterion $\mathrm{E}$ epoxy-coated slides (Schott) using a GMS 417 arrayer (Affymetrix). The printing procedures were similar to those that we used to construct cDNA microarrays (Liang et al. 2002, 2003). Unused amine binding sites on the slide were blocked by immersing the microarray in a solution containing $1 \mathrm{M}$ Tris ( $\mathrm{pH} 9$ ), $50 \mathrm{mM}$ ethanolamine, and $0.1 \%$ sodium dodecyl sulfate (SDS), at $50^{\circ} \mathrm{C}$ for $20 \mathrm{~min}$. MicroRNA samples $(n=4)$ were fluorescently labeled through a poly(A) extension reaction using the mirVana microRNA labeling kit (Ambion). Labeled microRNAs were purified and hybridized with microRNA microarrays for $16 \mathrm{~h}$ at $42^{\circ} \mathrm{C}$. Hybridized microarrays were washed and scanned using a ScanArray confocal scanner.

\section{Analysis of microRNA microarray data}

MicroRNA microarray data were analyzed using algorithms similar to those that we previously developed for cDNA microarrays (Liang et al. 2002, 2003; Knoll et al. 2005). Briefly, a microRNA was considered detectable on an array if its fluorescent intensity was significantly above negative controls. Detectable microRNAs were considered "low quality" and discarded if the fluorescent intensity was not distinguishable from the spot's own local background. Fluorescent intensities from replicate spots within an array were averaged following background subtraction, and normalized globally to a total intensity of 100,000 units for each array. A microRNA was considered detectable in a kidney region if the mean of its background-subtracted, normalized fluorescent intensity across all samples was significant above $0(P<0.05)$ according to a one-tailed, unpaired t-test. A microRNA was considered differentially expressed between the renal cortex and the renal medulla if the $P$-value of a two-tailed, unpaired $t$-test was $<0.02$ and the difference in fluorescent intensity was $>253$ adjusted units. The threshold of 253 adjusted units corresponded to two times the average standard deviation of the adjusted intensities of all microRNAs.

\section{Measurement of individual microRNAs using modified real-time PCR}

Expression levels of individual microRNAs were quantified using modified real-time PCR with either Taqman (Applied Biosystems) or Sybr Green (Ambion) chemistry. The method was similar to conventional real-time PCR (Knoll et al. 2005; Liang and Pietrusz 2007), with modifications that allowed detection of the short, mature microRNAs. To avoid any bias introduced during the microRNA enrichment procedure, total RNA samples were used in these analyses, and 5S rRNA was used as an internal normalizer. In addition, two samples of each kidney region that were not used in the microarray analysis were included in the modified real-time PCR analysis $(n=6)$.

\section{Preparation and fluorescent labeling of tissue proteins}

Kidneys of male Sprague-Dawley rats $(n=4)$ weighing 250-300 g were flushed in situ with cold normal saline. Renal cortex and renal medulla tissue was collected as described above. A piece of renal cortex or medulla were weighed and suspended 1:8 (weight: volume) in a lysis buffer containing $8 \mathrm{M}$ urea, 4\% CHAPS, 2\%
Pharmalyte 3-10, $30 \mathrm{mM}$ Tris, $10 \mu \mathrm{L} / \mathrm{mL}$ protease inhibitor mix (GE Healthcare), $10 \mu \mathrm{L} / \mathrm{mL}$ nuclease mix (GE Healthcare), and 5 $\mathrm{mM} \mathrm{MgAC}$. $\mathrm{pH}$ was adjusted with $\mathrm{HCl}$ to 8.5 on ice. Glass tubes and pestles were cooled to $-20^{\circ} \mathrm{C}$ before use. Tissue samples were homogenized on ice by 200 strokes with the motor set at $30 \%$. The homogenate was sequentially centrifuged at $500 \mathrm{~g}$ for $5 \mathrm{~min}$, $5000 \mathrm{~g}$ for $5 \mathrm{~min}$, and $18,000 \mathrm{~g}$ for $50 \mathrm{~min}$. The supernatant was collected and cleaned using a Bio-Rad 2D cleanup kit. Protein concentrations were measured using Bio-Rad RC DC Protein Assay.

For DIGE analysis, $50 \mu \mathrm{g}$ of each sample were mixed with 1 $\mu \mathrm{l}(400 \mathrm{pmol})$ of CyDye DIGE Fluor minimal dye (GE Healthcare) working solution. For each pair of cortex and medulla samples, Cy3 was used for one sample and Cy5 for the other. The two fluorescent dyes were swapped for a total of four pairs of samples. After a 30-min incubation period on ice in the dark, $1 \mu \mathrm{l}$ of $10 \mathrm{mM}$ lysine was added to the samples, which were then mixed, spun briefly in a microcentrifuge, and left on ice in the dark for 10 min. For internal reference, a pooled sample was generated by combining $50 \mu \mathrm{g}$ of each protein sample, and this pool was labeled with a third fluorescent dye, Cy2.

Two-dimensional gel electrophoresis and fluorescent scanning

Bio-Rad Protean IEF Cell was used for the first dimension isoelectric focusing. The 24-cm, pH 3-10 nonlinear (NL) IPG dry strips, DeStreak Rehydration Solution, and pH 3-10 NL IPG buffer were from GE Healthcare. A pair of cortex and medulla samples (labeled with Cy3 and Cy5), together with the internal reference (labeled with Cy2), were pooled and mixed with $4.5 \mu \mathrm{L} \mathrm{pH} \mathrm{3-10}$ NL IPG buffer and DeStreak Rehydration Solution (GE Healthcare) to a final volume of $450 \mu \mathrm{L}$ containing a total of $150 \mu \mathrm{g}$ of protein. IPG dry strips were rehydrated at $20^{\circ} \mathrm{C}, 50 \mathrm{~V}$ for $12 \mathrm{~h}$, then focused at $100 \mathrm{~V}$ for $1 \mathrm{~h}, 500 \mathrm{~V}$ for $2 \mathrm{~h}, 1000 \mathrm{~V}$ for $1 \mathrm{~h}$, followed by gradient increases of voltage to $10,000 \mathrm{~V}$ over $3 \mathrm{~h}$, then $10,000 \mathrm{~V}$ for 6 or $7 \mathrm{~h}$ until the $\mathrm{Vh}$ reached 80,000 . The dry strips were stored at $-80^{\circ} \mathrm{C}$ after isoelectric focusing.

For the second dimension electrophoresis, the IPG dry strips were equilibrated first for $15 \mathrm{~min}$ in SDS equilibration buffer containing $0.5 \%$ DTT, then $15 \mathrm{~min}$ in the same buffer with $4.5 \%$ $(\mathrm{w} / \mathrm{v})$ iodoacetamide, and then loaded onto $12.5 \%$ vertical SDSPAGE slab gels (Jule Inc.). SDS-PAGE was carried out on Ettan DALTtwelve electrophoresis systems (GE Healthcare) with 5 $\mathrm{W} /$ gel for the first $30 \mathrm{~min}$, then $10 \mathrm{~W} /$ gel for $7 \mathrm{~h}$. The gels were kept from light during electrophoresis and scanned immediately after electrophoresis.

The fluorescent signal was acquired with a Typhoon Variable Mode Imager (GE Healthcare). All gels were scanned using the same laser power and photomultiplier tube parameters.

\section{Differential expression and protein identification}

DeCyder 2D software (version 6.5) was used for protein spot detection, differential in-gel analysis, and biological variation analysis. Default parameters were used with the exception that the anticipated spot number was set at 3000 . Criteria for differential expression were a $P$-value of $<0.05$, difference of $>1.5$-fold, and the appearance of the spot in at least three of the four gels.

Preparative gels were run for cortex and medulla samples separately using the same isoelectric focusing and SDS-PAGE conditions as analytical gels, except the sample loading was increased to $500 \mu \mathrm{g}$ of protein. The gels were fixed in $5 \%$ acetic acid, 5\% methanol solution for $30 \mathrm{~min}$ after the electrophoresis, stained with $0.02 \%$ Coomassie Blue R350 (GE Healthcare) overnight, and destained in 5\% acetic acid, 5\% methanol solution for $12 \mathrm{~h}$. The gels were scanned with a Typhoon Variable Mode Imager. A pick list was obtained by matching the preparative gels with the analytical gels using DeCyder software. 
Protein spots were picked and digested by an Ettan Spot Handling Workstation (GE Healthcare). The peptide mass spectra were acquired using a Voyager DE PRO mass spectrometer (Applied Biosystems). The spectra were analyzed using Voyager Data Explore (version 4.0.0.0) with the following parameters: correlation factor 0.7 for noise filtering, trypsin autolysis peaks 842.52 and 2211.1046 for calibration, and $\mathrm{H} /$ peptides for peak deisotoping. The resulting peak list was used in Mascot searches of the MSDB database with the following parameters: up to one missed cleavage allowed, trypsin digest, carbamidomethyl (C) fixed modification, oxidation $(\mathrm{M})$ as the variable modification, peptide tolerance at 0.05 , and monoisotopic. The score thresholds for acceptance were set to 63 for the rodent database or 53 for the rat database.

\section{Western blot}

Protein abundance was analyzed using Western blot as we described previously (Liang and Pietrusz 2007). Coomassie blue staining was used to normalize the abundance of specific proteins. Antibodies for copper-zinc containing superoxide dismutase (1:200), fumarase (1:500), and heterogeneous nuclear ribonucleoprotein K $(1: 5,000)$ were from Santa Cruz Biotechnology. The antibody for catalase $(1: 2,000)$ was from Sigma.

\section{Bioinformatic analysis of microRNA-target pairs}

Three algorithms were used to computationally predict targets of differentially expressed microRNAs (Vinther et al. 2006). They included miRanda (http://microrna.sanger.ac.uk/targets/v4/), PicTar (http://pictar.bio.nyu.edu/), and TargetScan 3.1 (http:// www.targetscan.org/). The computationally predicted microRNA-target pairs were downloaded to a local database. Standard rat or human gene symbols of differentially expressed proteins were used to search the downloaded database. A microRNAtarget pair was selected if the microRNA was enriched and the abundance of the target protein was lower in one kidney region compared with the other. Redundancies in which a binding site was listed more than once because of multiple Ensembl IDs were removed.

The proportion of reciprocally expressed pairs of microRNAs and proteins that were computationally predicted to be microRNA-target pairs $\left(\mathrm{P}_{\mathrm{r}}\right)$ was calculated as $\mathrm{P}_{\mathrm{r}}=n_{\mathrm{r}} / N$, where $n_{\mathrm{r}}$ was the number of reciprocally expressed pairs of microRNAs and proteins that were predicted to be microRNA-target pairs, and $\mathrm{N}$ was the total number of reciprocally expressed microRNA-protein pairs. Similarly, the proportion of coexpressed microRNAs and proteins that were computationally predicted to be microRNAtarget pairs $\left(\mathrm{P}_{\mathrm{c}}\right)$ was calculated. Coexpression was defined as enrichment of both microRNA and protein in the same kidney region. In addition, eight microRNAs were randomly selected from the 377 microRNAs on the array. Computationally predicted targets of the randomly selected microRNAs were retrieved. The proportion of random microRNA-enriched protein pairs that were computationally predicted to be microRNA-target pairs $\left(\mathrm{P}_{\mathrm{m}}\right)$ was calculated. A $Z$-test was performed to examine if the chance for reciprocal expression $\left(\mathrm{P}_{\mathrm{r}}\right)$ to match computationally predicted microRNA-target pairs was significantly different than that of coexpression $\left(\mathrm{P}_{\mathrm{c}}\right)$ or random microRNAs $\left(\mathrm{P}_{\mathrm{m}}\right)$. Chi-square test or Fisher exact test (Vinther et al. 2006) yielded similar results as $Z$-test.

\section{Cell culture and transfection}

HK-2, a human kidney epithelial cell line, was obtained from and cultured as suggested by ATCC (Manassas, VA). Pre-miR and antimiR reagents were from Ambion. Transfection of HK-2 cells with
anti-miR or pre-miR (50 $\mathrm{nM}$ ) was performed using Oligofectamine following the procedures that we previously used for small interfering RNA (Liang and Pietrusz 2007). Cell homogenate was prepared for Western blot analysis $48 \mathrm{~h}$ after transfection.

\section{Statistics}

Data were analyzed using student t-test except in those special cases described above. $P<0.05$ was considered significant. Data are shown as mean \pm SEM.

\section{Acknowledgments}

The study was supported by NIH R01 HL077263 (M.L.) and N01HV-28182 (A.S.G.).

\section{References}

Ambros, V. 2004. The functions of animal microRNAs. Nature 431: $350-355$.

Arthur, J.M., Thongboonkerd, V., Scherzer, J.A., Cai, J., Pierce, W.M. and Klein, J.B. 2002. Differential expression of proteins in renal cortex and medulla: a proteomic approach. Kidney Int. 62: $1314-1321$.

Bagga, S., Bracht, J., Hunter, S., Massirer, K., Holtz, J., Eachus, R., and Pasquinelli, A.E. 2005. Regulation by let-7 and lin-4 miRNAs results in target mRNA degradation. Cell 122: 553-563.

Bartel, D.P. 2004. MicroRNAs: Genomics, biogenesis, mechanism, and function. Cell 116: 281-297.

Couzin, J. 2002. Breakthrough of the year: Small RNAs make big splash. Science 298: 2296-2297.

Dennis, C. 2002. 2002 in context: Small RNAs: The genome's guiding hand? Nature 420: 732.

Farh, K.K., Grimson, A., Jan, C., Lewis, B.P., Johnston, W.K., Lim, L.P., Burge, C.B., and Bartel, D.P. 2005. The widespread impact of mammalian MicroRNAs on mRNA repression and evolution. Science 310: $1817-1821$.

Gonzalez-Flecha, B., Evelson, P., Sterin-Speziale, N., and Boveris, A. 1993. Hydrogen peroxide metabolism and oxidative stress in cortical, medullary and papillary zones of rat kidney. Biochim. Biophys. Acta 1157: 155-161.

He, L. and Hannon, G.J. 2004. MicroRNAs: Small RNAs with a big role in gene regulation. Nat. Rev. Genet. 5: 522-531.

Hoorn, E.J., Hoffert, J.D., and Knepper, M.A. 2005. Combined proteomics and pathways analysis of collecting duct reveals a protein regulatory network activated in vasopressin escape. J. Am. Soc. Nephrol. 16: 2852-2863.

John, B., Enright, A.J., Aravin, A., Tuschl, T., Sander, C., and Marks, D.S. 2004. Human microRNA targets. PLoS Biol. 2: e363. doi: 10.1371/journal.pbio.0020363.

Kato, M., Zhang, J., Wang, M., Lanting, L., Yuan, H., Rossi, J.J., and Natarajan, R. 2007. MicroRNA-192 in diabetic kidney glomeruli and its function in TGF-beta-induced collagen expression via inhibition of E-box repressors. Proc. Natl. Acad. Sci. 104: 3432-3437.

Kim, V.N. 2005. MicroRNA biogenesis: Coordinated cropping and dicing. Nat. Rev. Mol. Cell Biol. 6: 376-385.

Knoll, K.E., Pietrusz, J.L., and Liang, M. 2005. Tissue-specific transcriptome responses in rats with early streptozotocin-induced diabetes. Physiol. Genomics 21: 222-229.

Krutzfeldt, J., Poy, M.N., and Stoffel, M. 2006. Strategies to determine the biological function of microRNAs. Nat. Genet. 38: S14-S19.

Lewis, B.P., Burge, C.B., and Bartel, D.P. 2005. Conserved seed pairing, often flanked by adenosines, indicates that thousands of human genes are microRNA targets. Cell 120: $15-20$.

Liang, M. and Pietrusz, J.L. 2007. Thiol-related genes in diabetic complications: A novel protective role for endogenous thioredoxin 2. Arterioscler. Thromb. Vasc. Biol. 27: 77-83.

Liang, M., Yuan, B., Rute, E., Greene, A.S., Zou, A.P., Soares, P., Question, M.C., Slocum, G.D., Jacob, G.R., Cowley, H.J., et al. 2002. Renal medullary genes in salt-sensitive hypertension: A chromosomal substitution and cDNA microarray study. Physiol. Genomics 8: 139-149.

Liang, M., Yuan, B., Rute, E., Greene, A.S., Olivier, M., and Cowley Jr., A.W. 2003. Insights into Dahl salt-sensitive hypertension revealed by temporal patterns of renal medullary gene expression. Physiol. Genomics 12: 229-237.

Lim, L.P., Lau, N.C., Garrett-Engele, P., Grimson, A., Schelter, J.M.,

\section{Genome Research}

www.genome.org 
Castle, J., Bartel, D.P., Linsley, P.S., and Johnson, J.M. 2005. Microarray analysis shows that some microRNAs downregulate large numbers of target mRNAs. Nature 433: 769-773.

Liu, C.G., Calin, G.A., Meloon, B., Gamliel, N., Sevignani, C., Ferracin, M., Dumitru, C.D., Shimizu, M., Zupo, S., Dono, M., et al. 2004. An oligonucleotide microchip for genome-wide microRNA profiling in human and mouse tissues. Proc. Natl. Acad. Sci. 101: 9740-9744.

Morrison, J., Knoll, K., Hessner, M.J., and Liang, M. 2004. Effect of high glucose on gene expression in mesangial cells: Upregulation of the thiol pathway is an adaptational response. Physiol. Genomics 17: 271-282

Naraba, H. and Iwai, N. 2005. Assessment of the microRNA system in salt-sensitive hypertension. Hypertens. Res. 28: 819-826.

O'Donnell, K.A., Wentzel, E.A., Zeller, K.I., Dang, C.V., and Mendell, J.T. 2005. c-Myc-regulated microRNAs modulate E2F1 expression. Nature 435: $839-843$.

Rajewsky, N. 2006. microRNA target predictions in animals. Nat. Genet. 38: S8-13.

Sood, P., Krek, A., Zavolan, M., Macino, G., and Rajewsky, N. 2006. Cell-type-specific signatures of microRNAs on target mRNA expression. Proc. Natl. Acad. Sci. 103: 2746-2751.

Stark, A., Brennecke, J., Bushati, N., Russell, R.B., and Cohen, S.M. 2005. Animal microRNAs confer robustness to gene expression and have a significant impact on 3'UTR evolution. Cell 123: 1133-1146.

Taylor, N.E., Glocka, P., Liang, M., and Cowley Jr., A.W. 2006. NADPH oxidase in the renal medulla causes oxidative stress and contributes to salt-sensitive hypertension in Dahl S rats. Hypertension 47: 692-698.

Thomson, J.M., Parker, J., Perou, C.M., and Hammond, S.M. 2004. A custom microarray platform for analysis of microRNA gene expression. Nat. Methods 1: 47-53.

Tsang, J., Zhu, J., and van Oudenaarden, A. 2007. MicroRNA-mediated feedback and feedforward loops are recurrent network motifs in mammals. Mol. Cell 26: 753-767.

Vinther, J., Hedegaard, M.M., Gardner, P.P., Andersen, J.S., and Arctander, P. 2006. Identification of miRNA targets with stable isotope labeling by amino acids in cell culture. Nucleic Acids Res. 34: e107. doi: 10.1093/nar/gkl590.

Wang, X. and Wang, X. 2006. Systematic identification of microRNA functions by combining target prediction and expression profiling. Nucleic Acids Res. 34: 1646-1652.

Witzmann, F.A., Fultz, C.D., Grant, R.A., Wright, L.S., Kornguth, S.E., and Siegel, F.L. 1998. Differential expression of cytosolic proteins in the rat kidney cortex and medulla: Preliminary proteomics. Electrophoresis 19: 2491-2497.

Received April 9, 2007; accepted in revised form November 28, 2007. 


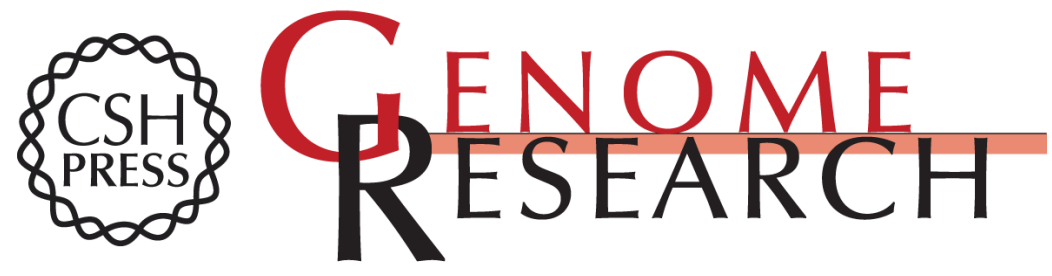

\section{MicroRNA-target pairs in the rat kidney identified by microRNA microarray, proteomic, and bioinformatic analysis}

Zhongmin Tian, Andrew S. Greene, Jennifer L. Pietrusz, et al.

Genome Res. 2008 18: 404-411 originally published online January 29, 2008

Access the most recent version at doi:10.1101/gr.6587008

Supplemental Material

References

License

Email Alerting Service
http://genome.cshlp.org/content/suppl/2008/01/31/gr.6587008.DC1

This article cites 33 articles, 8 of which can be accessed free at: http://genome.cshlp.org/content/18/3/404.full.html\#ref-list-1

Receive free email alerts when new articles cite this article - sign up in the box at the top right corner of the article or click here.

\section{Affordable, Accurate Sequencing.}

